\title{
Knowledge, attitudes, and practices regarding dengue infection among public sector healthcare providers in Machala, Ecuador
}

Andrew S. Handel ${ }^{1}$, Efraín Beltrán Ayala ${ }^{2,3}$, Mercy J. Borbor-Cordova ${ }^{4}$, Abigail G. Fessler ${ }^{5}$, Julia L. Finkelstein ${ }^{5}$, Roberto Xavier Robalino Espinoza ${ }^{3}$, Sadie J. Ryan ${ }^{6,7,8}$ and Anna M. Stewart-lbarra ${ }^{7 *}$

\begin{abstract}
Background: Dengue fever is a rapidly emerging infection throughout the tropics and subtropics with extensive public health burden. Adequate training of healthcare providers is crucial to reducing infection incidence through patient education and collaboration with public health authorities. We examined how public sector healthcare providers in a dengue-endemic region of Ecuador view and manage dengue infections, with a focus on the 2009 World Health Organization (WHO) Dengue Guidelines.
\end{abstract}

Methods: A 37-item questionnaire of dengue knowledge, attitudes, and practices was developed and administered to dengue healthcare providers in Machala, Ecuador. Survey focus areas included: "Demographics," "Infection and Prevention of Dengue," "Dengue Diagnosis and the WHO Dengue Guide," "Laboratory Testing," "Treatment of Dengue," and "Opinions Regarding Dengue."

Results: A total of 76 healthcare providers participated in this study, of which $82 \%$ were medical doctors and $14 \%$ were nurses. Fifty-eight percent of healthcare professionals practiced in ambulatory clinics and $34 \%$ worked in a hospital. Eighty-nine percent of respondents were familiar with the 2009 WHO Dengue Guidelines, and, within that group, 97 \% reported that the WHO Dengue Guide was helpful in dengue diagnosis and clinical management. Knowledge gaps identified included Aedes aegypti mosquito feeding habits and dengue epidemiology. Individuals with greater dengue-related knowledge were more likely to consider dengue a major health problem. Only $22 \%$ of respondents correctly reported that patients with comorbidities and dengue without warning signs require hospital admission, and $25 \%$ of providers reported never admitting patients with dengue to the hospital. Twenty percent of providers reported rarely ( $\leq 25 \%$ of cases) obtaining laboratory confirmation of dengue infection. Providers reported patient presumptive self-medication as an ongoing problem. Thirty-one percent of healthcare providers reported inadequate access to resources needed to diagnose and treat dengue.

Conclusion: Participants demonstrated a high level of knowledge of dengue symptoms and treatment, but additional training regarding prevention, diagnosis, and admission criteria is needed. Interventions should not only focus on increasing knowledge, but also encourage review of the WHO Dengue Guidelines, avoidance of presumptive self-medication, and recognition of dengue as a major health problem. This study provided an assessment tool that effectively captured healthcare providers' knowledge and identified critical gaps in practice.

Keywords: Dengue fever, KAP survey, Ecuador, Medical practitioners

\footnotetext{
*Correspondence: stewarta@upstate.edu

${ }^{7}$ Emerging Pathogens Institute, University of Florida, Gainesvillee, fl, USA

Full list of author information is available at the end of the article
} 


\section{Background}

Dengue virus infection is a major cause of morbidity, mortality, and economic hardship in the tropics and subtropics [1, 2]. Infection occurs when one of four dengue virus serotypes (DENV 1-4) are transmitted to humans by Aedes sp. (primarily Aedes aegypti) mosquitoes [1]. Dengue infection may cause fever, headache, abdominal pain, rash, muscle aches, and bone pain (hence 'break-bone fever'). Infection with additional dengue serotypes increases the risk of hemorrhagic disease, resulting in severe mucosal and gastrointestinal bleeding, hypovolemia, and potentially death [1]. It is crucial that healthcare professionals are able to accurately diagnose, monitor, treat, and hospitalize patients infected with dengue fever.

Latin America has seen a surge of dengue infections since the 1980s, increasing the need for physicians skilled in managing dengue. From 2010 to 2014, an average of 1.5 million cases per year were reported in the Americas [3], although total case estimates are higher due to underreporting [2]. Díaz-Quijano et al. [4] estimated that dengue-related mortality rates have tripled every decade in Latin America since dengue became endemic in the 1980s. The economic burden of dengue fever is also tremendous: the estimated median cost of dengue treatment in the Americas is US\$472 per ambulatory case (72.9\% of cases) and US\$1,227 per hospitalized case [5]. The total economic impact of dengue in the Americas was estimated at US\$2.1 billion per year (2000-2007 estimate; range US\$1-4 billion) [5], underscoring the significant economic burden of dengue fever infection and the need to improve interventions.

Understanding how clinicians manage suspected cases of dengue is crucial to improving patient outcomes. In 2009, the World Health Organization (WHO) revised its classification system of dengue severity [6]. The central aim of the new scheme is to improve clinical outcomes by identifying patients at highest risk of mortality who may require therapeutic interventions. However, acceptance and incorporation of these recommendations has varied considerably since publication, with ongoing debate regarding the utility of each classification scheme $[7,8]$. Recent investigations of the WHO Dengue Guidelines are promising. Prasad et al. [9] compared the sensitivity of the 2009 and 1997 WHO guidelines in identifying the severity of dengue infection among 56 patients who tested positive for dengue infection in northern India. The study found that, when compared to the 'gold standard' of actual level of medical intervention provided (i.e. outpatient versus inpatient treatment), the 2009 WHO classification system had $98.0 \%$ sensitivity, compared to $24.8 \%$ sensitivity using the 1997 system. In an analysis of 1,962 cases reviewed from 18 countries, Barniol et al. [10] found that $13.7 \%$ of cases could not be classified using the 1997 WHO classification system, compared to $1.6 \%$ using the 2009 WHO classification system. As the debate over dengue classification continues, it is critical to understand how clinicians interpret and apply the guidelines in clinical practice.

Previous research has focused on how community members view dengue infections; however, there have been few attempts to date to better understand the perspectives of clinicians. These studies have been conducted mainly in Asia [9, 11-15], with a single study performed in Puerto Rico [16]; to our knowledge, no studies to date have been conducted in Central or South America. Results have varied considerably across these studies. In a study of Sri Lankan practitioners [11], Kularatne et al. report significant disagreement among physicians over the utility of treating dengue with steroids, antibiotics, and platelet transfusions. Lee et al. [12] noted that clinical practice varied significantly by practice setting, as physicians practicing in private practice were more likely to refer patients with dengue to the hospital and to utilize dengue PCR testing (vs. serology), compared to physicians practicing at public clinics. Thaver et al. [13] conducted a knowledge-based assessment in Pakistan and found that practitioners had a stronger understanding of dengue pathophysiology than clinical diagnosis and treatment. Together, these studies provide evidence that clinical practice varies by region and over time, making it crucial to understand local, current practices for dengue management when identifying areas of potential improvement.

As the epidemiology of dengue has evolved over the past century, so have healthcare systems' strategies to reduce infection rates. Healthcare providers who interact directly with patients have an important role in both treating and preventing the spread of dengue. This study was conducted to assess the knowledge, attitudes, and practices regarding dengue infection among healthcare providers in a dengue-endemic city in Ecuador. We also assessed familiarity with the 2010 Pan American Health Organization's (PAHO) Spanish translation [17] of the 2009 WHO Dengue Guidelines, and how these guidelines influenced their clinical practice, providing important information to help guide future interventions.

\section{Methods}

\section{Study site and study population}

We conducted a study of the knowledge, attitudes, and practices associated with dengue infection among healthcare providers practicing in Machala, Ecuador, from December, 2013 through December, 2014. Machala is an urban coastal city located in El Oro Province, Ecuador $\left(3.2667^{\circ} \mathrm{S}, 79.9667^{\circ} \mathrm{W}\right.$, altitude $6 \mathrm{~m}$, population 245,972 ), and has been well-described as hyper-endemic 
for dengue fever (DENV 1-4) [18, 19]. Over a five year period (2010 to 2014), 72,060 cases of dengue were reported in Ecuador, with an annual average of 14,412 cases [20]. This study is part of an ongoing collaboration with the Ministry of Health to strengthen dengue surveillance capacities, with the aim of studying public sector healthcare providers; private physicians were therefore not included in our study. The Ecuadorian Ministry of Health previously collaborated with the Pan American Health Organization (PAHO) to translate the 2009 WHO Dengue Guidelines into a 2010 Spanish version of the guidelines [17], which was distributed throughout Machala and serves as a focal point of our study.

Physicians and nurses were recruited as the study population because they serve as the frontline healthcare workers for diagnosis and treatment of dengue and other febrile illnesses. Healthcare providers in Machala include primary care providers working in local healthcare clinics (Centros de Salud) and tertiary care providers practicing in public and private hospitals, including emergency care physicians, hospitalists, and subspecialists. The public health system requires that individuals visit a single assigned Centro de Salud prior to referral to hospital subspecialists. These clinics provide care free of charge. Private clinics were not included in this study. It is common for Ecuadorians to view hospital care as superior to ambulatory clinics, leading some patients to seek primary care in the Emergency Department.

\section{Participant recruitment}

Two methods of recruitment were utilized in this study. Participants from the public health sector were recruited at dengue management training conferences in Machala, with survey distribution prior to the educational session. These trainings were conducted in collaboration with and sponsored by the Ecuadorian Ministry of Health and the Global Emerging Infections Surveillance and Response System (GEIS, a division of the United States Armed Forces Health Surveillance Center), with the goal of improving recognition of dengue infection and awareness of the World Health Organization's Clinical Manual of Dengue. These individuals were recruited for the training sessions as they play key roles in dengue management. The second form of recruitment involved visits to the Ministry of Health public health clinics and to the Teófilo Dávila Hospital, the reference hospital for the province of El Oro.

\section{Questionnaire development}

We developed a 37-item questionnaire, with the goal of evaluating the knowledge, attitudes, and practices associated with dengue infection among healthcare providers. Information regarding dengue infection was based on the World Health Organization's Clinical Manual of
Dengue, with a subset of questions on local dengue epidemiology based on peer-reviewed sources $[3,13$, 18]. The questionnaire comprised of the following sections: "Demographics," "Infection and Prevention of Dengue," "Dengue Diagnosis and the WHO Guide," "Laboratory Testing," "Treatment of Dengue," and "Opinions Regarding Dengue" (See Additional file 1: Appendice A1 for English and Additional file 2: Appendice A2 for Spanish versions of the survey instrument). The survey was piloted through face-to-face interviews with physicians in Machala prior to conducting the full study.

\section{Data analysis}

Survey responses were analyzed using R (Version 3.1.2). Descriptive statistics (e.g. means, medians, frequency distributions) were calculated. A Cumulative Knowledge Score (CKS) was calculated as an aggregate of all knowledge-based questions (See questions in Tables 2 and 3). Correct answers received one point and incorrect answers received zero points, for a maximum possible score of 14 points. Questions requiring participants to select multiple correct answer choices were given one point per correct answer selected. A Clinical Scenario Score (CSS) was similarly developed from three clinical questions, with a maximum score of three points (See Table 3). These same clinical questions were included in the CKS. Bivariate Pearson Correlations ( $r$ ) were conducted to assess whether the CSS and CKS were associated with awareness and/or support of WHO clinical guidelines, prior training, years of experience or number of patients treated, and region of medical practice. We also examined whether dengue risk perceptions were associated with support for the WHO dengue guidelines, and the proportion of patients referred for dengue laboratory testing or hospital admission. The questions were grouped by dependent variable, and a Bonferroni correction was used for multiple comparisons. The alpha level was set at 0.05 (i.e., values of $p<0.05$ were considered statistically significant).

Closed-ended questions using a Likert scale and openended questions were used to assess doctor and patient perceptions of dengue (See Tables 5, 6 and 7). The frequencies of these themes were tabulated, and for each theme, and the average scores from the Likert scale were used to identify themes that associated with greater risk perceptions.

\section{Results/Discussion}

In this study, several common themes emerged; healthcare providers reported:

1. High use and awareness of the 2009 WHO Dengue Management Guidelines. 
2. High level of knowledge regarding dengue signs and symptoms, but demonstrated significant knowledge gaps regarding dengue epidemiology and prevention.

3. Limited knowledge of WHO-recommended criteria for dengue hospital admission, and under-utilization of confirmatory laboratory tests.

4. High level of concern regarding the burden of dengue in Machala; and a lack of training and basic tools needed to adequately diagnose and manage dengue infections.

5. High levels of presumptive self-medication and delay in seeking medical attention among patients with dengue fever.

This study assessed the knowledge, attitudes and practices of local healthcare providers in dengue management. This study was restricted to one group of healthcare practitioners in Machala at one point in time, and accordingly, the small sample size of available providers within Machala may limit generalizability of findings. Additionally, data collected were self-reported, limiting our ability to assess healthcare practices and causal inference. However, this study captures useful information from a community with a high burden of dengue, and this assessment framework can inform dengue management in other settings.

\section{Demographics}

A total of 76 healthcare providers involved in dengue care and treatment in Machala, Ecuador, participated in the study. Demographic information is presented in Table 1. Surveys were administered to participants during visits to their offices or at training events, resulting in a $100 \%$ response rate. Forty-one percent of participants were male and $59 \%$ were female. Ages ranged from under 30 years old to less than 70 years old, with a median age group of 41 to 50 years old and a median of 10 to 14 years of healthcare experience. Participants consisted of physicians (82\%), nurses (14\%), and other healthcare professionals $(4 \%)$. There are 93 physicians working in the public health sector in Machala including 63 doctors at 17 public health clinics, 15 in the central hospital, and 15 in the social security hospital, giving an inclusion rate of $67 \%$ of all potential physician subjects in the city. Healthcare providers worked primarily in public health clinics $(58 \%)$ and the Teófilo Dávila Hospital (34\%).

\section{Healthcare provider views of dengue burden}

As seen in Table 2, healthcare providers in Machala were concerned with dengue infections, with $89 \%$ of participants agreeing that it is a "major problem for my patient population". Of those in agreement, the majority reported that dengue is a significant threat because the
Table 1 Characteristics of Study Participants $(n=76)$

\begin{tabular}{|c|c|c|}
\hline Category & Response selected & $n(\%)$ \\
\hline \multirow[t]{2}{*}{ Gender } & Male & $31(41 \%)$ \\
\hline & Female & $45(59 \%)$ \\
\hline \multirow[t]{6}{*}{ Age (years) } & $<30$ & $24(32 \%)$ \\
\hline & $31-40$ & $10(14 \%)$ \\
\hline & $41-50$ & $17(23 \%)$ \\
\hline & $51-60$ & $17(23 \%)$ \\
\hline & $61-70$ & $6(8 \%)$ \\
\hline & $>70$ & $0(0 \%)$ \\
\hline \multirow[t]{3}{*}{ Medical role } & Doctor & $62(82 \%)$ \\
\hline & Nurse & $11(14 \%)$ \\
\hline & Other & $3(4 \%)$ \\
\hline \multirow[t]{6}{*}{ Years of Medical experience } & $<1$ & $6(8 \%)$ \\
\hline & $1-4$ & $26(34 \%)$ \\
\hline & $5-9$ & $5(7 \%)$ \\
\hline & $10-14$ & $7(9 \%)$ \\
\hline & $15-19$ & $10(13 \%)$ \\
\hline & $>19$ & $22(29 \%)$ \\
\hline \multirow[t]{4}{*}{ Practice setting $(n=74)$} & $\begin{array}{l}\text { Community Health Center } \\
\text { (Subcentro de Salud) }\end{array}$ & $43(58 \%)$ \\
\hline & Hospital & $25(34 \%)$ \\
\hline & Diagnostic Laboratory & $2(3 \%)$ \\
\hline & Other & $4(5 \%)$ \\
\hline
\end{tabular}

virus is endemic to the region and has the potential to cause high morbidity. One participant reported that dengue may cause "the deterioration of the [individual], family and community health". The majority of respondents (78 \%) also agreed with the statement, "My patients feel that dengue infection is a major problem for their health," with $34 \%$ citing health complications and

Table 2 Physician responses to the statement "I think that dengue is a major problem for my patient population" $(n=71)$

\begin{tabular}{ll}
\hline Categorical responses & Open-ended responses \\
\hline $\begin{array}{l}\text { Agree or Strongly Agree } \\
n=63,88 \%)\end{array}$ & The region is an endemic zone \\
& Dengue has a high morbidity \\
& There is a lack of preventative measures \\
& Patients self-medicate \\
& There exists poor infrastructure \\
& Dengue poses a high risk to others \\
& There is a lack of education about dengue \\
& There is a lack of social consciousness \\
regarding dengue
\end{tabular}


mortality as the major concerns in the general population. Seventeen percent of participants suggested that public health measures within the city, including disease prevention efforts, local infrastructure, and education were inadequate for controlling disease transmission. A small subset $(6 \%)$ of providers reported that dengue is not a major problem because the region already has effective prevention and treatment interventions in place. A similar proportion of providers also felt that a dengue diagnosis creates an unnecessary sense of fear among patients.

\section{Providers' views of the community response to dengue and self-medication}

The majority ( $76 \%$ ) of healthcare providers perceived that patients exhibiting symptoms of dengue would seek attention at a healthcare facility. An equal proportion also reported that patients are aware of the steps needed to prevent dengue infection (See Tables 2, 3 and 4 for physician attitudes toward dengue); of those who agreed with this statement, $30 \%$ suggested that public health awareness campaigns were successful. For example, one participant reported, "due to constant [educational] campaigns, [patients] know to seek out medical help before they develop alarms signs".

Seventeen percent of providers reported that upon symptom onset, many patients "turn to self-medication and do not seek out professional help". Previous studies have reported that communities in the urban periphery, and particularly men, report self-medicating to treat dengue [21]. This tendency to self-medicate can result in greater dengue morbidity and mortality due to lack of clinical management, and has the potential to increase community susceptibility to other diseases by promoting bacterial resistance to over-prescribed antibiotics.

\section{Clinical scenario scores and cumulative knowledge scores} The Cumulative Knowledge Score analysis results are presented in Tables 5 and 6 (Table 6 consists of the

Table 3 Physician responses to the statement "My patients feel that dengue infection is a major problem for their health" $(n=68)$

\begin{tabular}{ll}
\hline Categorical responses & Open-ended responses \\
\hline $\begin{array}{l}\text { Agree or Strongly Agree } \\
(n=53,78 \%)\end{array}$ & Dengue has a high morbidity \\
& Dengue is considered an alarming diagnosis
\end{tabular}

Dengue decreases economic productivity

Dengue is difficult to detect

The environment is conducive to disease transmission

Dengue poses a high risk to others

Neutral $(n=7,10 \%)$

Adequate medical attention is available

Disagree or Strongly

Disagree $(n=8,12 \%)$

Patients believe self-medication is adequate

There is a lack of education about dengue
Table 4 Physician responses to the statement "In my experience, a member of the community who exhibits dengue symptoms will seek medical attention" $(n=71)$

\begin{tabular}{ll}
\hline Categorical responses & Open-ended responses \\
\hline $\begin{array}{l}\text { Agree or Strongly Agree } \\
(n=54,76 \%)\end{array}$ & $\begin{array}{l}\text { Patients believe that dengue has a high } \\
\text { morbidity if untreated } \\
\text { Dengue symptoms are severe } \\
\text { Patients want to prevent complications } \\
\text { There is adequate education about dengue } \\
\text { There is easy access to medical attention }\end{array}$ \\
Neutral $(n=7,10 \%)$ & $\begin{array}{l}\text { Some patients will seek attention while others } \\
\text { self-medicate }\end{array}$ \\
Disagree or Strongly & $\begin{array}{l}\text { Patients do not seek medical attention until } \\
\text { complications develop }\end{array}$ \\
Disagree $(n=10,14 \%)$ & Medical care is delayed by self-treatment \\
\hline
\end{tabular}

Clinical Scenario subset of questions). The mean Cumulative Knowledge Score was 10.5 of 14 possible points (SD \pm 1.73$)$. Using a Bonferroni correction, the statistical significance level for CKS was determined to be $p<0.01$. The Cumulative Knowledge Score correlated positively with: 1) reporting familiarity with the WHO Dengue Guide ( $r=0.427, p<0.01), 2$ ) agreeing with the statement "I believe that dengue is a major problem for my patient population" ( $r=0.433, p<0.01)$, and 3 ) agreeing to the statement "My patients feel that dengue infection is a major problem for their health" $(r=0.282, p<0.01)$. Notably, having previous dengue training was not significantly correlated with the CKS $(p=0.225)$. These associations provide evidence of the interrelatedness of a practitioner's knowledge, patient care, and concern for dengue infection. Clinician education must not only focus on basic knowledge, but also emphasize dengue's burden on individual health and communities.

The Clinical Scenario Score analysis results are presented in Table 6. The mean Clinical Scenario Score was 2.1 of 3 potential points (Table 6). Using a Bonferroni correction, the statistical significance level for CSS was determined to be $p<0.0125$. A higher CSS was correlated with the following responses: 1 ) reporting familiarity with WHO Dengue Guidelines $(r=0.326, p<0.01), 2)$ agreeing with the statement "I believe that dengue is a major problem for my patient" $(r=0.37, p<0.01)$, 3) agreeing with the statement "I am fully trained to manage a patient with an infection of dengue without warning signs," ( $r=0.383, p<0.01)$, and 4$)$ agreeing with the statement "In my experience, a community member who has dengue symptoms will seek medical attention" ( $r=$ 0.453, $p<0.01)$. Higher CSS was also associated with reporting that the WHO Guidelines are helpful, although this was not statistically significant after a Bonferroni correction was applied $(r=0.245, p<0.05)$. These findings emphasize the importance of practitioner 'buy-in' of 
Table 5 Knowledge-Based Questions $(n=76)$

\begin{tabular}{lll}
\hline Question & Correct response & $n(\%)$ with correct response \\
\hline 1. How is dengue spread? & Aedes mosquito & $75(99 \%)$ \\
2. At what time of day are people most likely to be & Any answer other than "Night" & $57(75 \%)$
\end{tabular}

At what time of day are people most likely to be infected by dengue?

3. Which of the dengue serotypes have been found in Ecuador?

- Note: 0.25 point given per correct answer, with a total of DENV 1

$54(71 \%)$

1 point available

DENV 2

$54(71 \%)$

DENV 3

$48(63 \%)$

DENV 4

$34(45 \%)$

4. What advice do you give your patients to prevent dengue infection?

- Note: Question is worth a total of 2 points

1. Frequently change the water in flower vases

$66(87 \%)$

2. Remove containers that accumulate clean water

$69(91 \%)$

- 0.25 point given per correct answer, with a maximum of 1 point. Column to the right indicates the $n(\%)$ of respondents receiving

- 1 point given for not selecting "Take Paracetamol"

3. Eliminate tanks or puddles with stagnant water

$55(72 \%)$

4. Keep drinking water containers (cisterns, tanks) tightly closed

Did not select "Take Paracetamol"

$67(88 \%)$

$54(71 \%)$

5. Which group of patients should be hospitalized?

1. Dengue without warning signs (F)

$76(100 \%)$ a total of 4 points

2. Dengue without warning signs but with comorbidities (T)

$17(22 \%)$

- If the answer is correctly selected, the respondent gains 1 point

- If the answer is correctly left blank, the respondent gains 1 point

- Responses that are correct are marked here as $(T)$ and if incorrect are marked as (F)

6. According to the WHO's 2010 Clinical Management of Dengue guidebook, what signs and symptoms can be used to identify an infection of dengue without alarm signs?

- Note: Question is worth 1 point

3. Dengue with warning signs (T)

$59(78 \%)$

4. Severe dengue (T)

$58(76 \%)$

Percent answering all 4 correctly

$15(20 \%)$

Correct Responses

$n$ (\%) selecting response

- Each response is worth 1/19 point, which is given for either correctly selecting a true response or correctly leaving a false response blank

Headache

$59(78 \%)$

Muscle pain

60 (79\%)

Retro-orbital pain

$62(82 \%)$

Positive tourniquet test

$45(59 \%)$

Fever/subjective warmth

$64(84 \%)$

Petechial rash

$33(43 \%)$

Vomit

$25(33 \%)$

Incorrect

Ascites

$1(1 \%)$

Constipation

$5(7 \%)$

Diarrhea

Dyspnea

$3(4 \%)$

Dysuria

$2(3 \%)$

Chest pain

1 (1\%)

Edema

$2(3 \%)$

Icterus

$1(1 \%)$

Lymphadenitis

$3(4 \%)$

Nasal secretions

$11(14 \%)$

Persistent cough

$3(4 \%)$

Thrombocytopenia

15 (22\%)

Oral Hydration

$70(92 \%)$ 
Table 5 Knowledge-Based Questions ( $n=76$ ) (Continued)

7. Select any the treatments you could use in a patient suspected to have dengue

- Note: Question is worth 1 point

- 0.5 points given for hydration (either oral and/or IV) and

0.5 points given for paracetamol. Recipient is given 0 points if anti-bacterial or anti-viral medication is selected

\author{
IV Hydration \\ $12(16 \%)$ \\ $71(93 \%)$ \\ Paracetamol \\ $1(1 \%)$ \\ $1(1 \%)$ \\ Anti-viral \\ $0(0 \%)$ \\ Any of the following (listed individually in survey): Aspirin, \\ NSAIDs/Steroids/Immunosuppressants (methotrexate, \\ cyclosporine, etc.)/Opioids/Platelets/Plasma/Whole blood \\ transfusion
}

Note: One point given per question, unless otherwise specified

dengue's detrimental impact, as clinical knowledge and concern for dengue infections are strongly associated. Of note, the clinical scenarios comprised a small component (3 of 14 points) of the above-mentioned Cumulative Knowledge Score.

\section{Providing patients with accurate dengue prevention and treatment guidance}

Although study participants demonstrated a high level of understanding of dengue infection signs, symptoms, and treatment, we identified specific gaps in knowledge of dengue prevention and epidemiology. A total of $29 \%$ of participants incorrectly selected "take paracetamol" as a method for preventing dengue infection (Table 5). Although it is possible that some participants misinterpreted this question as asking which medications may help manage dengue, the survey clearly asked how dengue may be prevented, indicating a misconception of prevention strategies. In addition, $25 \%$ incorrectly selected "night time" as the most likely feeding time for Aedes mosquitoes. Similar findings have been documented elsewhere: Huang et al. [14] found that only $14.4 \%$ of Taiwanese providers correctly identified Aedes mosquito feeding habits, compared to $82.8 \%$ who correctly identified Anopheles mosquito feeding habits. When participants were asked which dengue virus serotypes are found in Ecuador, only 38 \% correctly answered all four serotypes (DENV 1-4). Ho [15] also found limited knowledge of dengue epidemiology among healthcare providers in Taiwan, with only $47.7 \%$ correctly responding that dengue is endemic in that country. These

Table 6 Clinical Knowledge Questions

\begin{tabular}{|c|c|c|}
\hline Question & Response Selected & $n(\%)$ \\
\hline \multirow{7}{*}{$\begin{array}{l}\text { 1. An 8-year old male patient presents to your office with a } \\
4 \text { day history of fever, nausea, vomiting three times per day, } \\
\text { and joint aches. He is accompanied by his mother, who } \\
\text { reports that he has been less active over the past few days } \\
\text { and seems to be getting more uncomfortable. You note the } \\
\text { following abnormalities on physical exam: The patient has } \\
\text { bleeding of the oral mucosa, a palpable mass on the right side } \\
2 \mathrm{~cm} \text { below the ribs, and winces when you palpate his } \\
\text { abdomen. You do not observe fluid in the abdomen or } \\
\text { difficulty breathing. Based on current WHO guidelines, this } \\
\text { patient is best classified as: }\end{array}$} & $(n=73)$ & \\
\hline & Dengue fever & $0(0 \%)$ \\
\hline & Dengue hemorrhagic fever & $5(7 \%)$ \\
\hline & Dengue shock syndrome & $0(0 \%)$ \\
\hline & Dengue without warning signs & $2(3 \%)$ \\
\hline & Dengue with warning signs ( $T$ ) & $61(83 \%)$ \\
\hline & Severe dengue & $5(7 \%)$ \\
\hline \multirow{4}{*}{$\begin{array}{l}\text { 2. A 5-year-old girl patient presents to your office with a few } \\
\text { days of fever and a distended, painful abdomen. Her mother } \\
\text { states that she has been less active over the past } 3 \text { days. It is } \\
\text { currently February and you have seen six patients in the past } \\
3 \text { weeks with dengue infections. The best course of action in } \\
\text { managing this patient is to: }\end{array}$} & $(n=73)$ & \\
\hline & $\begin{array}{l}\text { Order dengue lab tests, tell the patient to get rest at home, } \\
\text { and ask the patient to return to your office in } 24 \mathrm{~h}\end{array}$ & $10(14 \%)$ \\
\hline & $\begin{array}{l}\text { Order dengue lab tests and admit the patient to the hospital } \\
\text { for } 24 \mathrm{~h} \text { of observation (T) }\end{array}$ & $54(74 \%)$ \\
\hline & $\begin{array}{l}\text { Order dengue lab tests and admit the patient to the Intensive } \\
\text { Care Unit for close monitoring and access to emergency care }\end{array}$ & $9(12 \%)$ \\
\hline \multirow{4}{*}{$\begin{array}{l}\text { 3. A 27-year-old male patient presents to your office in February } \\
\text { with two days of fever and complaints of muscle aches. He } \\
\text { notes that he has had three episodes of non-bloody vomiting } \\
\text { in the past two days. The patient notes that his younger sister } \\
\text { has similar symptoms. You recall hearing numerous reports of } \\
\text { dengue infection during the last month. The best course of } \\
\text { action in managing this patient is to: }\end{array}$} & $(n=71)$ & \\
\hline & $\begin{array}{l}\text { Order dengue lab tests, tell the patient to get rest at home, } \\
\text { and ask the patient to return to your office in } 24 \mathrm{~h}(\mathrm{~T})\end{array}$ & $52(73 \%)$ \\
\hline & $\begin{array}{l}\text { Order dengue lab tests and admit the patient to the hospital } \\
\text { for } 24 \mathrm{~h} \text { of observation }\end{array}$ & $19(27 \%)$ \\
\hline & $\begin{array}{l}\text { Order dengue lab tests and admit the patient to the ICU for } \\
\text { close monitoring and access to emergency care }\end{array}$ & $0(0 \%)$ \\
\hline
\end{tabular}


misunderstandings may lead healthcare providers to give patients incorrect, clinically significant advice. It is critical to target specific local misconceptions of dengue prevention and transmission through training of medical professionals, in order to reduce the burden of dengue.

\section{Confusion regarding hospital admission criteria}

Clinicians indicated confusion when developing appropriate dengue treatment plans for their patients. When healthcare professionals were asked which groups of patients with dengue require hospital admission, only $22 \%$ correctly stated that patients with "dengue without warning signs but with comorbidities" require hospital admission (Table 5). The 2009 WHO Dengue Guidelines provide specific recommendations for appropriate clinical observation based on a patient's risk of significant morbidity [7]. These guidelines state that any patient with a comorbidity (e.g. diabetes mellitus, obesity, risk of hemorrhage such as peptic ulcer disease) should be admitted to a hospital during a dengue infection, regardless of the severity of infection. Additionally, only $45 \%$ of participants correctly responded to all three clinical scenarios (Table 6), demonstrating knowledge gaps of patient admission criteria.

Hospital admission rates for dengue infection vary considerably between regions globally. For example, Tomashek et al. [16] found that only $31 \%$ of Puerto Rican medical providers used hospital admission criteria consistent with the 1997 WHO Dengue Guidelines. Conversely, Lee et al. [12] reported that one-third of providers in Singapore "always" or "often" admitted patients with suspected dengue, regardless of infection severity. Globally, it is estimated that less than $5 \%$ of patients infected with dengue will develop severe disease [22], and WHO recommends that patients who do not meet criteria for hospitalization have frequent office follow-up [7]. This is particularly important in resource-limited settings. Patients with comorbidities who are not admitted to hospitals may have worse clinical outcomes, underscoring the need for close monitoring of this patient population.

\section{Diagnostic testing: under-utilization and inadequate resources}

Study participants indicated suboptimal use of confirmatory diagnostic laboratory tests when dengue infection was suspected (Table 7). As appropriate in a region with many acute febrile illnesses with similar clinical presentations as dengue, $61 \%$ of healthcare providers reported referring all patients with suspected dengue infection for laboratory test confirmation. However, $20 \%$ of participants reported referring patients for confirmatory laboratory tests $25 \%$ of the time or less. As these patients may actually be infected with other febrile illnesses such as leptospirosis, malaria, or chikungunya, laboratory confirmation is crucial for differential diagnosis and to
Table 7 Practice-Based Questions

\begin{tabular}{|c|c|c|}
\hline Question & Response selected & $n(\%)$ \\
\hline \multirow{5}{*}{$\begin{array}{l}\text { Approximately how many patients do } \\
\text { you see per week? }(n=60)\end{array}$} & 0 & $3(5 \%)$ \\
\hline & $1-49$ & $6(10 \%)$ \\
\hline & $50-99$ & $\begin{array}{l}28 \\
(46.7 \%)\end{array}$ \\
\hline & $100-149$ & $\begin{array}{l}16 \\
(26.7 \%)\end{array}$ \\
\hline & $>150$ & 7 (11.7 \%) \\
\hline \multirow{2}{*}{$\begin{array}{l}\text { Are you familiar with the WHO's } 2010 \\
\text { Clinical Management of Dengue } \\
\text { guidelines? }\end{array}$} & Yes & 67 (89\%) \\
\hline & No & $8(11 \%)$ \\
\hline \multirow{2}{*}{$\begin{array}{l}\text { Do you feel that the WHO's Dengue } \\
\text { guidelines help in managing dengue? }\end{array}$} & Yes & $64(97 \%)$ \\
\hline & No & $2(3 \%)$ \\
\hline \multirow{6}{*}{$\begin{array}{l}\text { Of those patients who you suspect } \\
\text { have dengue fever, approximately } \\
\text { what percentage do you refer to a lab } \\
\text { for diagnostic testing? }\end{array}$} & $0 \%$ of patients & 1 (1 \%) \\
\hline & $10 \%$ of patients & $10(15 \%)$ \\
\hline & $25 \%$ of patients & $3(4 \%)$ \\
\hline & $50 \%$ of patients & $8(12 \%)$ \\
\hline & $75 \%$ of patients & $5(7 \%)$ \\
\hline & $100 \%$ of patients & $40(61 \%)$ \\
\hline \multirow{2}{*}{$\begin{array}{l}\text { Do your patients ever use a private lab } \\
\text { without a referral? }\end{array}$} & Yes & $31(47 \%)$ \\
\hline & No & $35(53 \%)$ \\
\hline \multirow{6}{*}{$\begin{array}{l}\text { Approximately what percentage of } \\
\text { patients with dengue fever do you } \\
\text { refer to the hospital for additional } \\
\text { medical treatment? }\end{array}$} & 0 & $14(26 \%)$ \\
\hline & $<10 \%$ & $31(57 \%)$ \\
\hline & $25 \%$ & 1 (2 \%) \\
\hline & $50 \%$ & $6(11 \%)$ \\
\hline & $75 \%$ & 1 (2 \%) \\
\hline & $100 \%$ & $1(2 \%)$ \\
\hline \multirow{2}{*}{$\begin{array}{l}\text { Do you feel you have adequate } \\
\text { resources to treat your patients when } \\
\text { they have dengue? }\end{array}$} & Yes & 48 (69 \%) \\
\hline & No & $22(31 \%)$ \\
\hline \multirow{4}{*}{$\begin{array}{l}\text { If you said 'No' to the previous } \\
\text { question, what are you lacking? } \\
\text { - Note: Percentages given as n/22, } \\
\text { based on previous question } \\
\text { - Note: Subjects may select multiple } \\
\text { options }\end{array}$} & Sufficient training & 7 (32%) \\
\hline & $\begin{array}{l}\text { Medication needed } \\
\text { to treat }\end{array}$ & $9(41 \%)$ \\
\hline & $\begin{array}{l}\text { Instruments needed } \\
\text { to treat }\end{array}$ & $8(36 \%)$ \\
\hline & Access to lab tools & $10(45 \%)$ \\
\hline
\end{tabular}

Note: Percentages given do not include respondents who did not answer the question

inform appropriate medical interventions. It is important to note that $14 \%$ of providers reported inadequate access to diagnostic testing for dengue (Table 7). Additionally, providers who agreed with the statement "I am fully trained to manage a patient with an infection of dengue without warning signs" referred a higher percentage of their patients for laboratory testing $(r=0.345, p<0.01)$, compared to those who disagreed with this statement. This may signal one of two possibilities: clinical confidence is increased with better access to diagnostic 
testing, or providers who report greater confidence in their clinical training refer more patients for confirmatory laboratory tests. Access to dengue diagnostic testing remains a key issue in this context.

Healthcare providers were asked about availability and access to a variety of resources for dengue diagnosis and treatment. A total of $31 \%$ of providers reported having inadequate resources (See Table 7 for specific resources). There were no significant correlations between reported lack of resources for dengue diagnosis and treatment and insufficient training, Cumulative Knowledge Scores, treatments used, or other items from this survey. Identifying the impact of resource deficiencies is difficult to assess from the data collected, as no discernible differences in knowledge, attitudes, or practices were identified in this study. Further investigation of availability and access to resources for dengue diagnosis and treatment, and how they influence daily clinical practice is needed.

\section{Awareness and Implementation of the WHO dengue guidelines}

Awareness of the 2009 WHO Dengue Guidelines was high, with $89 \%$ of participants reporting previous knowledge of the guidelines. Of these respondents, $97 \%$ reported that these guidelines were helpful. This finding is in contrast to Kularatne's study of Sri Lankan practitioners [13], in which only $45 \%$ of practitioners reported using the WHO Dengue Guidelines. However, Kularatne's study was conducted prior to the current version of the WHO Dengue Guidelines, and may be more related to local medical practices and training.

\section{Impact of practice setting}

In this study, there were no significant differences in reported knowledge, attitudes, and practice, between healthcare providers practicing in a hospital versus ambulatory settings, including familiarity with the 2009 WHO Dengue Guidelines, reporting that these guidelines were helpful, or overall dengue knowledge $(p>0.05)$. Previous studies have indicated that practice settings can influence clinical management of dengue fever. Ho et al. [15] found that healthcare providers practicing at Taiwanese medical centers (i.e. medical school-affiliated hospitals at the highest accreditation level) had significantly different levels of knowledge, compared to providers at non-medical centers. In order for dengue interventions to be most effective in hyper-endemic regions, healthcare providers of all types and at all settings must receive adequate training and guidance, and differences in knowledge, attitudes, and practice by setting should continue to be assessed.

\section{Conclusion}

Findings from this study provide important insights into medical practitioner knowledge, attitudes, and practices associated with dengue fever in a resource-limited endemic region. These findings highlight several strategies to improve diagnosis and clinical management of dengue infections in this region. A strong healthcare policy begins with accurate information, which can best be obtained and disseminated through close collaboration between the public, primary healthcare providers, health educators, and the public health sector.

- Healthcare providers should receive continuous education about dengue prevention, transmission, and high-risk patient populations.

- Providers' needs should be assessed in future studies, as nearly one-third of participants reported inadequate access to crucial healthcare resources.

- Health providers should educate their patient population about the harms of self-diagnosis and presumptive self-medication.

- Findings demonstrated that those providers who showed the greatest concern of dengue infections were also the most knowledgeable and provided clinical care that more closely aligned with WHO recommendations. Future interventions should therefore provide core dengue information while emphasizing dengue's impact on health and development.

- Periodic reassessment of the local knowledge, attitudes, and clinical practices will be instrumental to reduce the burden of dengue fever and improve clinical management in high-burden settings.

\section{Additional files}

Additional file 1: Appendix A1: Knowledge, Attitudes, and Practices of Dengue Survey - English Version. (DOC 49 kb)

Additional file 2: Appendix A2: Knowledge, Attitudes and Practices of Dengue Survey - Spanish Version. (DOC 55 kb)

\section{Abbreviations}

CKS, Cumulative Knowledge Score; CSS, Clinical Scenario Score; PAHO, Pan American Health Organization; WHO, World Health Organization

\section{Acknowledgments}

Thank you to the healthcare providers of Machala for their participation and to SNEM Machala, MSP Ecuador, and the Center for Global Health and

Translational Science at SUNY Upstate Medical University for their support.

\section{Funding}

The IDSA Medical Scholars Program provided a stipend to ASH to support this study.

Availability of data materials

Survey instruments in English and Spanish are included as supplements. Raw survey data available upon request.

Authors' contributions

ASH was involved in study design, creation of the questionnaire, data collection, statistical analysis, interpretation of results, and coordinating and drafting the manuscript. EBA was involved in study design, creation of the 
questionnaire, and data collection. MJBC was involved in study design, creation of the questionnaire, and interpretation of results. AGF participated in statistical analysis and interpretation of results. JLF participated in interpretation of results and review of the manuscript draft. RXRE was involved in study design, creation of the questionnaire, and data collection. SJR was involved in study design, creation of the questionnaire, interpretation of results, and review of the manuscript. AMSI conceived of the study, and was involved in study design, creation of the questionnaire, data collection, statistical analysis, interpretation of results, and coordinating and drafting the manuscript.

\section{Competing interests}

The authors declare that they have no competing interests with the research.

\section{Consent for publication}

N/A

\section{Ethics approval and consent to participate}

The investigation protocol was reviewed and approved by the Institutional Review Boards (IRB) of the State University of New York (SUNY) Upstate Medical University in Syracuse, New York, and the Ecuadorian Ministry of Health. The study was certified as exempt by the IRBs (as all participants were over the age of 18 and no personal identifying information was collected), and no informed consent was required during the administration of the questionnaire.

\section{Author details}

'Department of Pediatrics, Stony Brook Children's Hospital, Stony Brook, NY USA. ${ }^{2}$ Universidad Técnica de Machala, Machala, Ecuador. ${ }^{3}$ Ministerio de Salud Publica, Machala, El Oro, Ecuador. ${ }^{4}$ Facultad de Ingenieria Maritima, Ciencias Oceanicas y Recursos Naturales, Escuela Superior Politécnica del Litoral (ESPOL), Guayaquil, Guayas, Ecuador. ${ }^{5}$ Division of Nutritional Sciences, Cornell University, Ithaca, NY, USA. ${ }^{6}$ Department of Geography, University of Florida, Gainesville, FL, USA. ${ }^{7}$ Emerging Pathogens Institute, University of Florida, Gainesvillee, fl, USA. ${ }^{8}$ Center for Global Health and Translational Science, State University of New York Upstate Medical University, Syracuse, NY, USA.

Received: 2 February 2016 Accepted: 25 May 2016

\section{Published online: 01 June 2016}

\section{References}

1. Halstead S. Dengue. Lancet. 2007:370(9599):1644-52

2. Hotez PJ, Bottazzi ME, Franco-Paredes C, Ault SK, Periago MR. The neglected tropical diseases of Latin America and the Caribbean: a review of disease burden and distribution and a roadmap for control and elimination. PLoS Negl Trop Dis. 2008:2(9):e300.

3. $\mathrm{PAHO}$ (n.d.) Number of reported cases of dengue and severe dengue (DS) in the Americas by country (2010-2014). Washington: Pan American Health Organization. http://www.paho.org/hq/index.php?option=com_ topics\&view=readall\&cid=3273\&ltemid=40734\&lang=en. Accessed $27 \mathrm{Sep}$ 2015.

4. Díaz-Quijano FA, Waldman EA. Factors associated with dengue mortality in Latin America and the Caribbean, 1995-2009: an ecological study. Am J Trop Med Hyg. 2012;86(2):328-34

5. Shepard DS, Coudeville L, Halasa YA, Zambrano B, Dayan GH. Economic impact of dengue illness in the Americas. Am J Trop Med Hyg. 2011;84(2): 200-7.

6. World Health Organization. Dengue: guidelines for diagnosis, treatment, prevention and control. Geneva: World Health Organization; 2009.

7. Farrar JJ, Hien TT, Hortisck O, Hung NT, Jaenisch T, Junghanns T, et al. Dogma in classifying dengue disease. Am J Trop Med Hyg. 2013;89(2):198-201.

8. Halstead SB. Dengue: the syndromic basis to pathogenesis research. Inutility of the 2009 WHO case definition. Am J Trop Med Hyg. 2013;88(2):212-5.

9. Prasad D, Kumar C, Jain A, Kumar R. Accuracy and applicability of the revised WHO classification (2009) of dengue in children seen at a tertiary healthcare facility in Northern India. Infection. 2013:41(4):775-82.

10. Barniol J, Gaczkowski R, Barbato EV, da Cunha RV, Salgado D, Martínez E, et al. Usefulness and applicability of the revised dengue case classification by disease: multi-centre study in 18 countries. BMC Infect Dis. 2011;11:106.
11. Kularatne SA. Survey on the management of dengue infection in Sri Lanka: opinions of physicians and pediatricians. Southeast Asian J Trop Med Public Health. 2005;36(5):1198-200.

12. Lee LK, Thein TL, Kurukularatne C, Gan VC, Lye DC, Leo YS. Dengue knowledge, attitudes, and practices among primary care physicians in Singapore. Ann Acad Med Singapore. 2011;40(12):533-8.

13. Thaver AM, Sobani ZA, Qazi F, Khan M, Zafar A, Beg MA. Assessing the need for training: general practitioners' knowledge, attitude and practice concerning dengue and malaria in Karachi, Pakistan. Int Health. 2011;3(2): 126-30.

14. Huang HL, Chiu TY, Huang KC, Cheng SY, Yao CA, Lee LT. Travel-related mosquito-transmitted disease questionnaire survey among health professionals in Taiwan. J Travel Med. 2011;18(1):34-8.

15. Ho TS, Huang MC, Wang SM, Hsu HC, Liu CC. Knowledge, attitude, and practice of dengue disease among healthcare professionals in Southern Taiwan. J Formos Med Assoc. 2013;112(1):18-23.

16. Tomashek KM, Biggerstaff BJ, Ramos MM, Pérez-Guerra CL, Garcia Rivera EJ, Sun W. Physician survey to determine how dengue is diagnosed, treated and reported in Puerto Rico. PLoS Negl Trop Dis. 2014;8(10):e3192.

17. PAHOMHO. Manejo Clínico Del Dengue - Dengue Diagnóstico Y Tratamiento De Casos. 2010. http://www.paho.org/ecu/index.php?option=com_ content\&view=article\&id=544: materiales-enfrentar-dengue\&catid=692:ecu.13comunicacin-social\&ltemid=289. Accessed 27 Sep 2015.

18. Stewart-lbarra AM, Ryan SJ, Belt Beltrán E, Mejía R, Silva M, Muñoz A. Dengue vector dynamics (Aedes Aegypti) influenced by climate and socia factors in Ecuador: implications for targeted control. PLoS One. 2013;8(11): e78263.

19. Stewart-lbarra AM, Lowe R. Climate and non-climate drivers of dengue epidemics in Southern Coastal Ecuador. Am J Trop Med Hyg. 2013;88(5): 971-81.

20. Anuario Epidemiologico. 1994-2014. Direccion Nacional de Vigilancia Epidemiologica, Ministerio de Salud Publica de Ecuador. http://www.salud.gob. ec/direccion-nacional-de-vigilancia-epidemiologica/. Accessed 1 Apr 2016.

21. Stewart-Ibarra AM, Luzadis VA, Borbor-Cordova MJ, Silva M, Ordoñez T, Beltrán E, Ryan SJ. A social-ecological analysis of community perceptions of dengue fever and Aedes aegypti in Machala, Ecuador. BMC Public Health. 2014;14(1):1135.

22. Tomashek KM, Sharp RM, Morgolis HS. Chapter 3: Infectious Diseases Related to Travel: Dengue. In: CDC Health Information for International Travel. New York: Oxford University Press; 2014.

\section{Submit your next manuscript to BioMed Central and we will help you at every step:}

- We accept pre-submission inquiries

- Our selector tool helps you to find the most relevant journal

- We provide round the clock customer support

- Convenient online submission

- Thorough peer review

- Inclusion in PubMed and all major indexing services

- Maximum visibility for your research

Submit your manuscript at www.biomedcentral.com/submit
) Biomed Central 